\title{
Large scale ionization of the Radio Arc region by the Quintuplet and the Arches clusters ${ }^{\star}$
}

\author{
N. J. Rodríguez-Fernández, J. Martín-Pintado, and P. de Vicente
}

\begin{abstract}
Observatorio Astronómico Nacional, Instituto Geográfico Nacional, Apdo. 1143, 28800 Alcalá de Henares, Madrid, Spain
\end{abstract}

Received 11 May 2001 / Accepted 5 July 2001

\begin{abstract}
We present an analysis of selected fine structure lines (Ne II $12.8 \mu \mathrm{m}$, Ne III $15.6 \mu \mathrm{m}, \mathrm{S}$ III 18.7 and $33.5 \mu \mathrm{m}$, O III 52 and $88 \mu \mathrm{m}, \mathrm{N}$ II $122 \mu \mathrm{m}$ and N III $57 \mu \mathrm{m}$ ) observed with the Infrared Space Observatory (ISO) toward the Radio Arc in the Galactic center region (GCR). Most of the data were retrieved from the ISO Data Archive. We study the density of the ionized gas and the large scale ionization structure in a region of $\sim 30 \times 30 \mathrm{pc}^{2}$ by means of the O III $52 \mu \mathrm{m} / 88 \mu \mathrm{m}$, S III $18.7 \mu \mathrm{m} / 33.5 \mu \mathrm{m}$, Ne III $15.6 \mu \mathrm{m} / \mathrm{Ne}$ II $12.8 \mu \mathrm{m}$ and $\mathrm{N}$ III $57 \mu \mathrm{m} / \mathrm{N}$ II $122 \mu \mathrm{m}$ line ratios. The electron densities $\left(n_{\mathrm{e}}\right)$ derived from the O III lines ratio indicate the presence of diffuse ionized material with $n_{\mathrm{e}}$ of $\sim 10^{1.8-2.6} \mathrm{~cm}^{-3}$. The Ne III $15.6 \mu \mathrm{m} / \mathrm{Ne}$ II $12.8 \mu \mathrm{m}$ and $\mathrm{N}$ III $57 \mu \mathrm{m} / \mathrm{N}$ II $122 \mu \mathrm{m}$ line ratios vary from source to source from 0.05 to 0.30 and from 0.3 to 2.5 , respectively. The N III 57/N II $122 \mu \mathrm{m}$ ratio show two clear gradients, one pointing toward the Quintuplet cluster and the other pointing toward the Arches cluster. We have used a simple model to explain the ionization structure observed in the N III/N II and Ne III/Ne II lines ratios. The model shows that the large scale ionization of the whole region can be accounted for by the UV radiation produced by the Quintuplet and the Arches cluster. Any other ionization mechanism should play a minor role. We also investigate the influence of the clusters on the bubble of warm dust (hereafter Radio Arc Bubble, RAB) seen in the Midcourse Space Experiment (MSX) infrared images. We find that the warm dust is well correlated with the ionized gas indicating that the dust is also heated by the radiation from both clusters. Furthermore, the elliptical rather than circular symmetry of some structures like the Thermal Filaments can also be explained when one considers the combined effects of both the Arches and the Quintuplet clusters. We have also found that the RAB is filled with continuum emission of hard X-rays and with emission from the $6.4 \mathrm{keV}$ line of neutral or low ionized Fe. We briefly discuss the implications of these findings on the structure and morphology of the GCR interstellar medium and the possible origin of the RAB.
\end{abstract}

Key words. Galaxy: center - ISM: H II regions - ISM: dust, extinction - ISM: individual objects: Radio Arc infrared: ISM: lines and bands - X-rays: ISM: lines and bands

\section{Introduction}

With the aim of understanding the thermal balance and ionization of the interstellar medium (ISM) in the Galactic center region $(\mathrm{GCR})$, we have undertaken a systematic study at millimeter and infrared wavelengths of a sample of clouds distributed along the Nuclear Bulge (in notation of Mezger et al. 1996) and the "Clump 2" complex. These clouds exhibit large column densities of warm $\mathrm{H}_{2}$ (with temperatures of $\sim 150 \mathrm{~K}$ ) which constitute an important fraction of the total column densities of molecular gas (Rodríguez-Fernández et al. 2001). In addition, the comparison of fine-structure lines of several ionized atoms

Send offprint requests to: N. J. Rodríguez-Fernández, e-mail: nemesio@oan.es

* Based on observations with ISO, an ESA project with instruments funded by ESA Member States (especially the PI countries: France, Germany, the Netherlands and the United Kingdom) and with the participation of ISAS and NASA. with hydrogen radio recombination lines indicate that the GCR ISM should be rather inhomogeneous and that the the ionized gas could be distributed, for instance, in relatively low density ionized bubbles (Martín-Pintado et al. 2000a; Rodríguez-Fernández et al. in prep.).

The two sources of our sample with more intense fine structure lines $(\mathrm{M}+0.16-0.10$ and $\mathrm{M}+0.21-0.12$, see Martín-Pintado et al. 2000a) are located in the vicinity of the Radio Arc, which is one of the most prominent radiocontinuum features in the GCR. The Radio Arc, located at $l \sim 0^{\circ} 17$, is perpendicular to the Galactic plane and it is apparently connected to Sgr A by a "bridge" of radiocontinuum emission at positive galactic latitude (see e.g. Güsten 1989). VLA observations showed that the Radio Arc is composed by long and thin straight filaments that emit non-thermal radiation (Yusef-Zadeh et al. 1984; Yusef-Zadeh et al. 1987). These filaments are usually known as the Non-Thermal Filaments (NTFs) and indicate the presence of a strong poloidal component of 
the magnetic field in the GCR (Yusef-Zadeh et al. 1984; Yusef-Zadeh \& Morris 1987a, b).

On the other hand, the "bridge" is composed by a set of arched filaments that emit thermal radiation (Thermal or Arched Filaments). In the vicinity of the NTFs and probably interacting with them there are other prominent features that also emit thermal radiocontinuum. These are G0.18-0.04 (the Sickle), which is approximately located where the NTFs cross the galactic plane, and a gun-shaped source located south of the Sickle known as the Pistol Nebula (G0.15-0.05). The thermal nature of the Sickle, the Pistol Nebula and the Thermal filaments has been established by the association of the radiocontinuum emission with radio recombination line emission (Pauls et al. 1976; Paul \& Mezger 1980; Yusef-Zadeh et al. 1987).

In the recent years, the origin of the ionization of these thermal features has been a subject of great interest. It was first thought that the Sickle and the Thermal Filaments were the surfaces of molecular clouds ionized by collisions between the neutrals and the ions that spiral around the magnetic field lines associated with the NTFs (Yusef-Zadeh \& Morris 1987c; Morris \& Yusef-Zadeh 1989; Serabyn \& Güsten 1991; Serabyn \& Morris 1994). However, with the discovery of the outstanding stellar cluster known as the "Quintuplet" or AGFL 2004 (Nagata et al. 1990; Okuda et al. 1990; Glass et al. 1990) the effect of ultra-violet (UV) radiation on the ionization of the Sickle was revised. Nowadays, it is commonly assumed that this cluster is the main ionization source of the Sickle H II region (Timmermann et al. 1996; Simpson et al. 1997). Furthermore, there is increasing evidence that the Pistol Nebula is also ionized by the Quintuplet instead of by the Pistol star, located in the center of curvature of the nebula (Moneti et al. 1999; Figer et al. 1999).

On the other hand, the Object 17 in the infrared survey undertaken by Nagata et al. (1993) was found to be a cluster of young stars (Nagata et al. 1995; Cotera et al. 1996). This cluster, also known as G0.121+0.017 or the Arches cluster have enough OB stars to be the ionization source of the Thermal Filaments (Cotera et al. 1996; Serabyn et al. 1998). However, to explain the homogeneity in the excitation observed in the fine structure line emission, Colgan et al. (1996) proposed that more hot stars should be distributed rather uniformly through the region of the Arched Filaments. Nonetheless, the recent analysis of fine structure lines observed by ISO (Cotera et al. 2000a) and the recombination lines VLA observations of Lang et al. (2001), suggest that the Arches cluster alone could account for the ionization of the Arched Filaments.

Recently, mid-infrared images $(7-25 \mu \mathrm{m})$ of the GCR taken by the Midcourse Space Experiment (MSX) satellite have shown a new morphological feature in the Radio Arc region (Egan et al. 1998 ). It is a ring of warm dust which is also present, although it is rather weak, in the $20 \mathrm{~cm}$ radio image of Yusef-Zadeh \& Morris (1987c). Although it is not yet clear its actual morphology (bubble, ring or cylinder), in the following we will call it the Radio Arc
Bubble (RAB). The RAB is also seen in the IRAS HIRES maps (see Simpson et al. 1997) where it appears as a ring of warm dust emitting mainly at 25 and $60 \mu \mathrm{m}$. The PAHs emission detected by ISO in the RAB shows the presence of carbon-rich material in the bubble (Levine et al. 1999). The part of the Sickle which is perpendicular to the NTFs just lie in the north-west edge of the RAB, suggesting that the Sickle is part of this large structure of warm dust. The geometrical center of the RAB is located just on the most intense NTF at $(l, b) \sim\left(0.16,-0{ }^{\circ} 11\right)$, north-west of the non-thermal maximum (G0.16-0.15).

Figer et al. (1999) suggested that winds and radiation pressure from O-type stars in the Quintuplet cluster or even a supernova explosion cloud have blown the dust bubble. They proposed that the offset of the Quintuplet cluster from the center of the RAB could be due to the relative motion of the stellar cluster with respect to the ISM or to large-scale density fluctuations in the medium where the bubble is expanding. However, the nearly circular shape of the RAB seen in the radio images of Yusef-Zadeh \& Morris (1987c) rules out the hypothesis of a density gradient.

The purpose of this paper is to test the scenario of a very inhomogeneous medium - proposed by Martín-Pintado et al. (2000a) to explain the ionization of $\mathrm{M}+0.12-0.12$ and $\mathrm{M}+0.16-0.10$ - by studying the large scale ionization structure in the region of the Radio Arc. We have analyzed selected fine structure lines of the observations taken with the Infrared Space Observatory (ISO; Kessler et al. 1996) towards the Radio Arc. Previous works were restricted to the Sickle and the Pistol Nebula. We study the Sickle in the broader context of the RAB and investigate the possible connection between the ionization in the Radio Arc area and the existence of the RAB. The paper is organized as follows: Sect. 2 defines the sample of sources selected from the ISO Data Archive (IDA) and Sect. 3 comments on the data reduction and results. The analysis of the results is presented in Sect. 4 and, with the help of photoionization models, in Sect. 5. The results are discussed in Sect. 6 and 7. Finally, Sect. 8 summarizes the main conclusions.

\section{Observational data}

To study the large scale ionization structure of the Radio Arc region and its possible connection to the ring or bubble of warm dust (hereafter RAB) we have used Short Wavelength Spectrometer (SWS; de Graauw et al. 1996) and Long Wavelength Spectrometer (LWS; Clegg et al. 1996) ISO data of our sources $M+0.16-0.10$ and $\mathrm{M}+0.21-0.12$. We have also retrieved other LWS and SWS observations towards this region from the ISO Data Archive (IDA). We have analyzed the NeIII $15.6 \mu \mathrm{m}$, Ne II $12.8 \mu \mathrm{m}$ and the S III 33.5 and $18.7 \mu \mathrm{m}$ fine structure lines observed with the SWS. In the LWS observations we have analyzed the fine structure lines of N III at $57 \mu \mathrm{m}$, $\mathrm{N}$ II at $122 \mu \mathrm{m}$ and $\mathrm{O}$ III at 52 and $88 \mu \mathrm{m}$. Table 1 lists IDA identification numbers (TDTs), coordinates and observing 


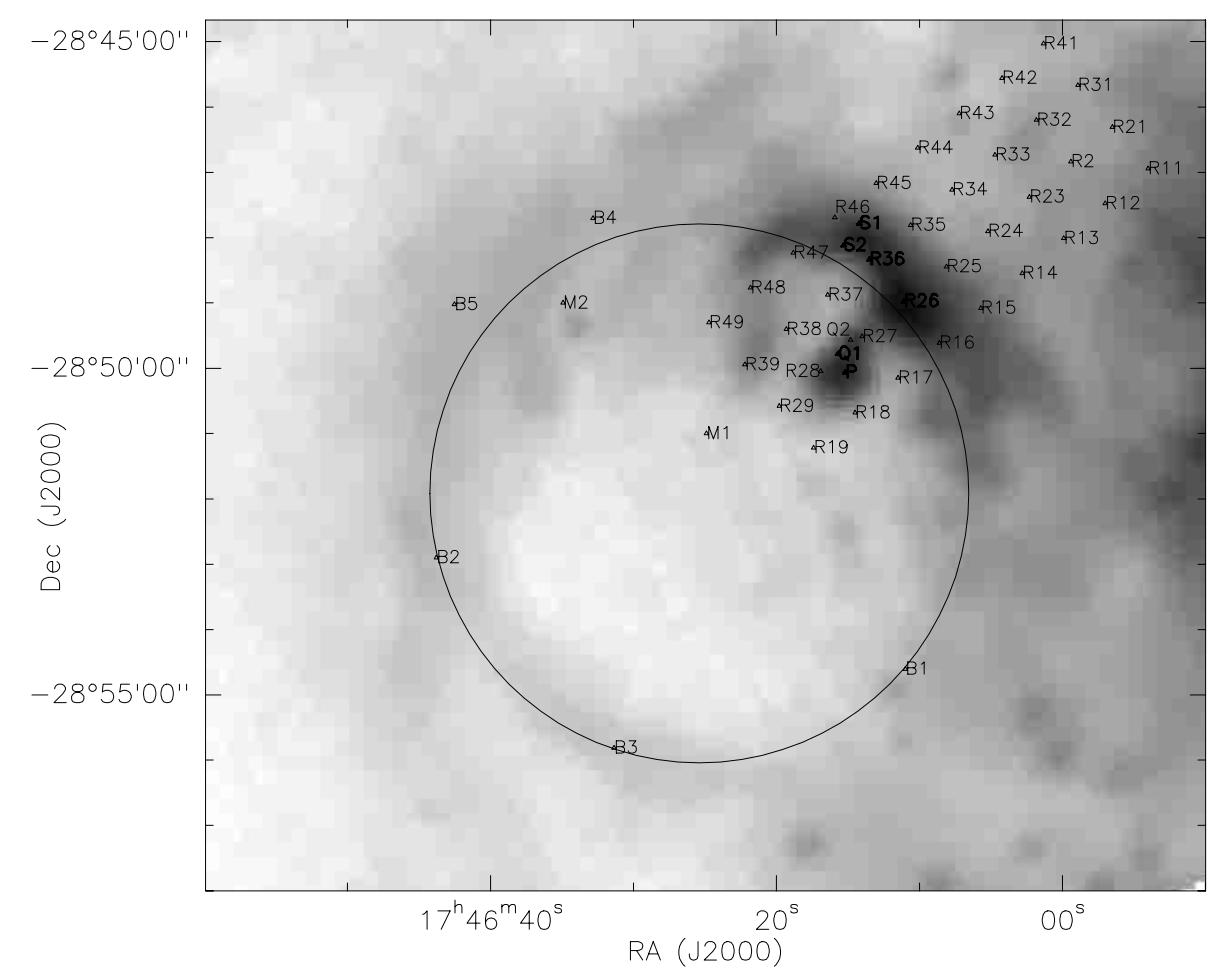

Fig. 1. Position of the sources observed by ISO SWS and LWS overlaid on the band $E(18.2-25.1 \mu \mathrm{m})$ MSX image. The display is logarithmic from $4 \times 10^{-6}$ to $8 \times 10^{-4} \mathrm{~W} \mathrm{~m}^{-2} \mathrm{sr}^{-1}$. The circle delineates the edges of the warm dust bubble (RAB, see also Fig.7). The strong features to the north-west of the RAB are the Sickle and the Pistol Nebula.

modes (AOTs), for all the selected sources. Figure 1 shows the position of the data points overlaid on the 18.2 $25.1 \mu \mathrm{m}$ band MSX image in which the RAB is clearly visible.

Positions M1 (M+0.16-0.10) and M2 (M+0.21-0.12) are the two clouds from our sample located in the Radio Arc region (Martín-Pintado et al. 2000a; RodríguezFernández et al. 2001). M1 lies close to the geometrical center of the RAB over the most intense NTF. Positions $\mathrm{B} 1$ to $\mathrm{B} 3$ lie in the southern part of the RAB (see Levine et al. 1999) while B4 and B5 are located in the northeast edge of the RAB, close to M2. Q1 and Q2 are the Quintuplet sources GCS3 II and GCS4 (see Chiar et al. 2000), S1 and S2 are two observations toward the Sickle and $\mathrm{P}$ toward the Pistol Nebula. $\mathrm{R}$ is a raster map of $4 \times 9$ points (4 rows, 9 columns), with a spacing of $50^{\prime \prime}$ and rotated $50^{\circ}$ north from west. In the following, individual points in the raster will be named as $\mathrm{R} x y$, i.e., $\mathrm{R} 11, \mathrm{R} 12, \ldots$

\section{Data reduction and results}

The observations consisted of SWS full grating spectra (SWS01), SWS grating spectra of selected lines (SWS02), and LWS full grating spectra (LWS01). The telescope aperture at the wavelengths of the lines of interest are listed in Tables 2 and 3. M1 and M2 have been processed through the Off Line Processing (OLP) version 7.0. The other observations have been automatically reprocessed when retrieval from the IDA with OLP versions 9.1 to 9.5. Further reduction and analysis have been carried out with ISAP 2.0 .
Table 1. Observations summary.

\begin{tabular}{lllll}
\hline Position & RA $^{\mathrm{a}}$ & Dec $^{\mathrm{a}}$ & TDT $^{\mathrm{b}}$ & AOT $^{\mathrm{c}}$ \\
\hline M1 & $17: 46: 24.9$ & $-28: 51: 00.0$ & 48502207 & SWS 02 \\
& & & 48502309 & LWS 01 \\
M2 & $17: 46: 34.9$ & $-28: 49: 00.0$ & 48502612 & SWS 02 \\
& & & 49401613 & LWS 01 \\
$\mathrm{R}^{\mathrm{d}}$ & $17: 46: 09.4$ & $-28: 48: 07.4$ & 67700702 & LWS 01 \\
B1 & $17: 46: 11.0$ & $-28: 54: 36.3$ & 46301403 & SWS 01 \\
B2 & $17: 46: 43.8$ & $-28: 52: 53.9$ & 46300901 & SWS 01 \\
B3 & $17: 46: 31.4$ & $-28: 55: 48.8$ & 49800804 & SWS 01 \\
B4 & $17: 46: 32.8$ & $-28: 47: 42.2$ & 69601107 & LWS 01 \\
B5 & $17: 46: 42.5$ & $-28: 49: 01.3$ & 69601107 & LWS 01 \\
Q1 & $17: 46: 15.7$ & $-28: 49: 47.0$ & 29702147 & SWS 01 \\
Q2 & $17: 46: 14.8$ & $-28: 49: 34.0$ & 28701246 & SWS 01 \\
S1 & $17: 46: 14.2$ & $-28: 47: 47.1$ & 67700503 & SWS 01 \\
S2 & $17: 46: 15.4$ & $-28: 48: 07.0$ & 46400904 & SWS 02 \\
P & $17: 46: 15.2$ & $-28: 50: 04.0$ & 84101302 & SWS 01 \\
\hline
\end{tabular}

a J2000 coordinates.

b Observation number.

c Astronomical Observing Template (instrument and observing mode).

d $4 \times 9$ raster map. The coordinates are the center of the raster.

\subsection{SWS AOTs 01 and 02}

As a first stage, we have removed bad data points. Afterwards, for the AOT 01 observations, we have shifted the different detectors to a common level and we have 
Table 2. Fluxes derived from Gaussian fits to the lines observed with the SWS in units of $10^{-20} \mathrm{~W} \mathrm{~cm}^{-2}$. Numbers in parentheses are the rms errors of the last significant digit.

\begin{tabular}{lllll}
\hline & Ne II & Ne III & S III & S III \\
$\lambda(\mu \mathrm{m})$ & 12.81 & 15.55 & 18.71 & 33.48 \\
Beam $\left(\operatorname{arcsec}^{2}\right)$ & $14 \times 27$ & $14 \times 27$ & $14 \times 27$ & $20 \times 33$ \\
\hline M1 & $41(2)$ & $9.3(13)$ & $14.4(10)$ & $102(3)$ \\
M2 & $139(2)$ & $20(2)$ & $63(2)$ & $425(12)$ \\
B1 & $92(8)$ & $19(2)$ & $41(2)$ & $236(8)$ \\
B2 & $179(5)$ & $30(4)$ & $80(4)$ & $640(30)$ \\
B3 & $138(12)$ & $10(4)^{\mathrm{a}}$ & $56(3)$ & $480(20)$ \\
Q1 & $439(12)$ & $149(6)$ & $270(6)$ & $600(20)$ \\
Q2 & $122(12)$ & $35(12)$ & $267(6)$ & $373(13)$ \\
S1 & $610(30)$ & $125(11)$ & $340(20)$ & $1860(90)$ \\
S2 & $491(5)$ & $90(2)$ & - & - \\
P & $500(29)$ & $227(6)$ & $319(8)$ & $879(14)$ \\
\hline
\end{tabular}

a Low signal-to-noise ratio $(\sim 3)$.

averaged across scan directions and detectors. For the AOT 02 observations we have averaged across scan directions before shifting the different detectors to a common level and averaging across detectors. Baselines of order 1 were removed from the spectra. The fluxes of the lines with their corresponding rms errors as derived from Gaussian fits are listed in Table 2. Errors in the line fluxes due to calibration uncertainties are smaller than $25 \%$ for the Ne III and Ne II lines and less than $20 \%$ and $30 \%$ for the S III 18.7 and $33.4 \mu \mathrm{m}$, respectively (Leech et al. 2001).

\subsection{LWS AOT 01}

For all the LWS01 observations, detector SW2 showed memory effects at wavelengths shorter than $55 \mu \mathrm{m}$. To analyze the O III $52 \mu \mathrm{m}$ line we have treated the two scan directions independently, averaging only across scans. For both scan directions the fluxes are in agreement within 15\%. The fluxes of the O III $52 \mu \mathrm{m}$ lines listed in Table 3 are the average of the fluxes derived independently for the two scans directions. For the lines at other wavelengths we have shifted the different scans to a common level before averaging across the two scan directions and across scans. We have subtracted from the spectra baselines of order 1 . The line fluxes and rms errors as derived from the Gaussian fits are listed in Table 3.

The O III $88 \mu \mathrm{m}$ line is in the overlap region of detectors SW5 and LW1. We have analyzed both detectors separately and the measured fluxes agree within $15 \%$. The fluxes listed in Table 3 are the average values of both determinations. Before fitting Gaussian profiles to the O III $88 \mu \mathrm{m}$ (detector LW1) and the N II $122 \mu \mathrm{m}$ (detector LW3) lines we have defringed the spectra. The line flux calibration uncertainties are expected to be smaller than $30 \%$ (Swinyard et al. 1996).

The raster map, $\mathrm{R}$, has been reduced using the same procedure described above and Fig. 2 shows the emission maps for all the lines. The maximum of the emission is found towards the Sickle for all but the N II $122 \mu \mathrm{m}$ line, which peaks in the western part of the map where the intensity of the N III line also increases. The emission, however, does not completely follow the Sickle. The part of the Sickle parallel to the NTFs that points toward the center of the RAB is not clearly seen in any of the emission maps. The N III map also shows a local maximum at the position of the Pistol Nebula.

\subsection{Comparison with previous observations}

Some observations presented in this paper have also been previously carried out with other instruments. One can compare the LWS O III 52 and $88 \mu \mathrm{m}$ and N III $57 \mu \mathrm{m}$ lines fluxes in the Sickle region with those measured by Simpson et al. (1997). Their points P2, P5, P3, P4, P8 and P6 are included in the LWS raster map. Assuming extended homogeneous emission and taking into account the differences in the beams sizes of both observations, one finds that for the sources located in the Sickle (P2, P5, P3 and P4) the fluxes measured by Simpson et al. are $60 \%$ higher than those obtained with the LWS. On the other hand, for P8 and P6 they are rather similar. These results indicate that the emission is indeed quite homogeneous in the area of their P8 and P6 positions (approximately corresponding to $\mathrm{R} 34$ and $\mathrm{R} 28$ respectively) but not in the region of the Sickle. However, even in the Sickle region, the clumpiness of the fine structure line emission in the LWS beam is relatively small and the approximation of extended and homogeneous emission is rather good (see also Martín-Pintado et al. 2000a). A similar conclusion is derived if one compares our O III $88 \mu \mathrm{m}$ and $\mathrm{N}$ III $57 \mu \mathrm{m}$ data with the observations of the Sickle by Timmermann et al. (1996). Taking into account that they had a beam of $22^{\prime \prime}$ and assuming homogeneous emission, the expected flux in the LWS beam $\left(\sim 80^{\prime \prime}\right)$ is larger than that observed for some points and lower for other points being on average $17 \%$ larger than those measured with the LWS. Given the different beam sizes and the flux calibration uncertainties, we conclude that the LWS data are in good agreement with previous data and that the fine structure lines observed with the LWS show a relatively uniform emission.

\section{Analysis}

\subsection{Extinction correction}

Before undertaking further analysis of the data it is necessary to correct them for the dust extinction effects. For the typical average visual extinction of $\sim 25$ mag measured towards the GCR (Catchpole et al. 1990; Schultheis et al. 1999) even at mid and far IR wavelengths the extinction corrections are non negligible. We can use different methods to estimate the extinction affecting our data. One possibility is to use the ratio of the S III lines at 18.7 and $33.5 \mu \mathrm{m}$ to derive lower limits to the extinction for the sources where these two lines are available. This is 


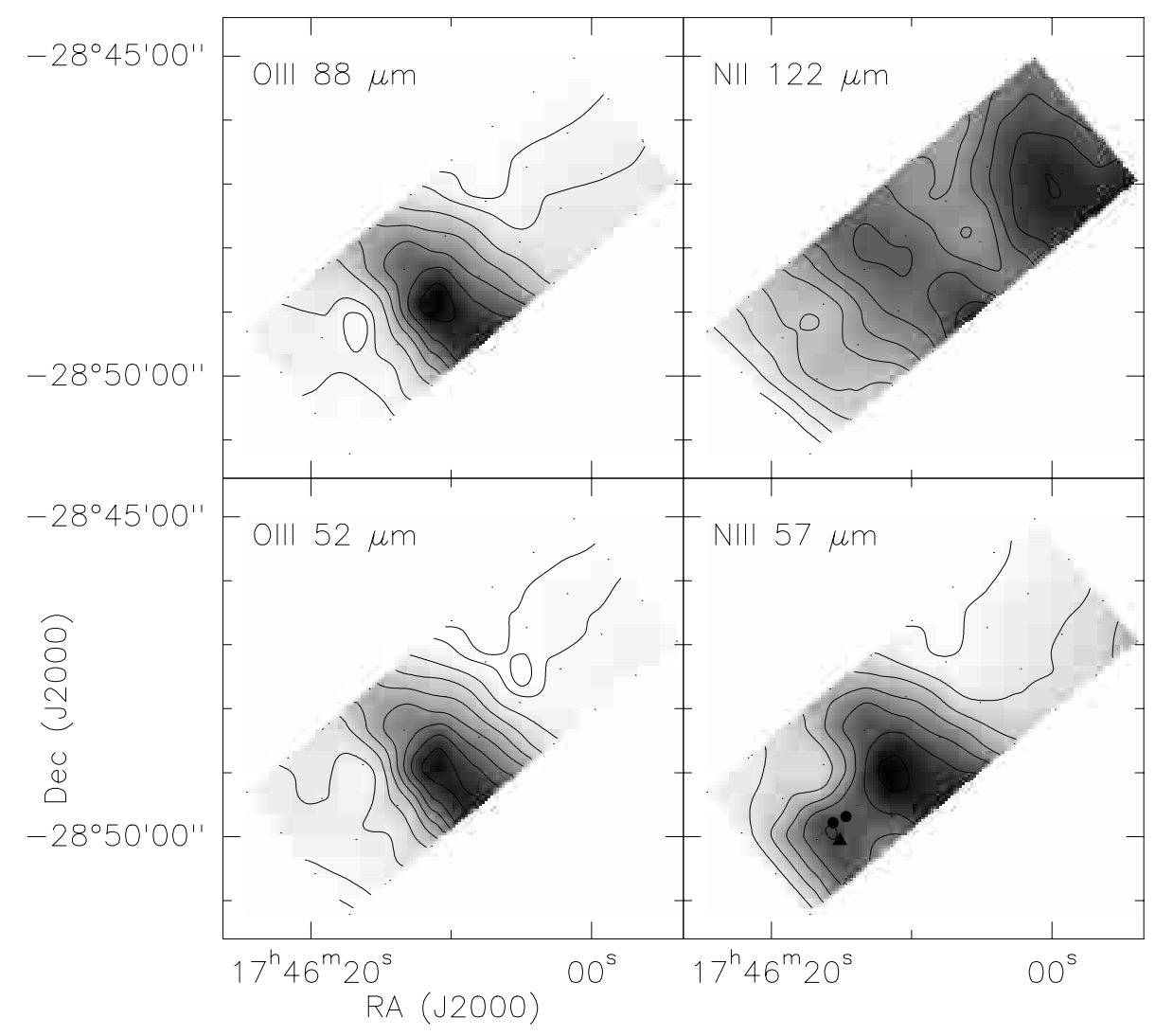

Fig. 2. LWS raster maps. Upper-left: O III $88 \mu \mathrm{m}$ map (contour levels in units of $10^{-18} \mathrm{~W} \mathrm{~cm}^{-2}: 6,10$, and from 12.5 to 42.5 by steps of 6). Lower-left: O III $52 \mu \mathrm{m}$ map (contour levels in units of $10^{-18} \mathrm{~W} \mathrm{~cm}^{-2}$ : 7, 9, and from 14 to 50 by steps of 6 ). Upper-right: N II $122 \mu \mathrm{m}$ map (contour levels in units of $10^{-18} \mathrm{~W} \mathrm{~cm}^{-2}$ : from 4 to 15 by steps of 1 ). Lower-right: N III $57 \mu \mathrm{m}$ map (contour levels in units of $10^{-18} \mathrm{~W} \mathrm{~cm}^{-2}$ : from 3.8 to 21.8 by steps of 3 ). The strong elongated feature corresponds to the Sickle for all but the N II $122 \mu \mathrm{m}$ map. The filled circles shown in the N III $57 \mu \mathrm{m}$ map indicate the location of the Quintuplet sources GCS 4 and GCS 3II and the triangle indicate the position of the Pistol Nebula.

Table 3. Fluxes derived from Gaussian fits to the lines observed with the LWS. Fluxes in units of $10^{-19} \mathrm{~W}^{\mathrm{cm}^{-2}}$. Numbers in parentheses are the rms errors of the last significant digit.

\begin{tabular}{lllll}
\hline & O III & N III & O III & N II \\
$\lambda(\mu \mathrm{m})$ & 51.81 & 57.33 & 88.36 & 121.90 \\
Beam $\left(\operatorname{arcsec}^{2}\right)$ & $80 \times 77$ & $80 \times 77$ & $84 \times 76$ & $78 \times 75$ \\
\hline M1 & $50(3)$ & $23(4)$ & $65(5)$ & $26(2)$ \\
M2 & $108(5)$ & $48(3)$ & $118(4)$ & $74(5)$ \\
B4 & $124(10)$ & $59(6)$ & $147(8)$ & $92(6)$ \\
B5 & $71(8)$ & $38(6)$ & $102(10)$ & $90(6)$ \\
R & $52(7)-518(20)^{\mathrm{a}}$ & $29(3)-223(10)^{\mathrm{a}}$ & $67(6)-441(9)^{\mathrm{a}}$ & $40(3)-140(4)^{\mathrm{a}}$ \\
\hline
\end{tabular}

${ }^{a}$ Maximum and minimum values in the raster. See also Fig. 2.

because the S III $18.7 / 33.5$ ratio cannot be lower than the theoretical limit of 0.5 for the case of very low density. If the measured ratio is lower than the predicted lower limit, this indicates a higher extinction for the S III $18.7 \mu \mathrm{m}$ than for the S III $33.5 \mu \mathrm{m}$ line. From our S III data, we derived a lower limit to the visual extinction assuming extended emission for all the positions of $\sim 20$ mag at most.

A direct determination for the overall extinction in the area of the Sickle has been recently made by
Cotera et al. (2000b) both by analyzing the stellar content and by comparing the radiocontinuum emission with the $\mathrm{Br} \gamma$ emission. They found an extinction at $2.2 \mu \mathrm{m}$ of $\sim 2.5-3.2 \mathrm{mag}$. These values imply a visual extinction of $\sim 25-32 \mathrm{mag}$, in agreement with the large scale studies of Catchpole et al. and Schulteis et al. and consistent with our lower limits. In the following we will assume that our data is affected by an average visual extinction of 30 mag. To extrapolate the visual extinction to the wavelengths of 
the SWS lines we have used the extinction law derived by Lutz (1999) towards Sgr A*. This extinction law is almost equivalent to that of Draine (1989) at the wavelengths of the lines presented in this paper. To derive the extinction for the LWS lines we have used the following extinction law:

$$
A_{\lambda}=0.014 A_{\mathrm{V}}(30 / \lambda)^{1.5}
$$

where $\lambda$ is the wavelength in microns, $A_{\lambda}$ is the extinction at that wavelength, and $A_{\mathrm{V}}$ is the visual extinction.

\subsection{Electron densities}

Table 4 lists the extinction corrected O III 52 to $88 \mu \mathrm{m}$ and S III 18.7 to $33.5 \mu \mathrm{m}$ line intensity ratios (hereafter O III 52/88 and S III 18/33 ratios, respectively) and Fig. 3b shows the map of the extinction corrected O III $52 / 88$ ratio for the raster. The most prominent feature in this map is a ridge that goes northeast-southwest at the position of the Pistol Nebula. The O III $52 / 88 \mu \mathrm{m}$ ratio in the map ranges between 0.78 and 1.43. Table 4 also list the electron densities $\left(n_{\mathrm{e}}\right)$ derived from these ratios assuming that the lines are excited by collisions with electrons and that the sources are extended (see Rubin et al. 1994). The derived O III $52 / 88$ ratio implies $n_{\mathrm{e}}$ between $10^{1.8}$ and $10^{2.6} \mathrm{~cm}^{-3}$. From the S III 18/33 ratio we can only derive an upper limit to $n_{\mathrm{e}}$ of $\sim 100 \mathrm{~cm}^{-3}$ for most sources. For the Pistol Nebula and the Quintuplet sources (P, Q1 and Q2) the derived densities from the S III lines are as high as $10^{3.2-3.8} \mathrm{~cm}^{-3}$. These densities are higher than those derived for the same region from the O III lines (with a larger beam), suggesting the presence of small scale structures with high density in the Sickle and the Pistol Nebula as previously mentioned in Sect. 3.3.

\subsection{Ionization structure}

Table 4 lists the extinction corrected N III 57 to N II $122 \mu \mathrm{m}$ and Ne III 15.5 to Ne II $12.8 \mu \mathrm{m}$ ratios (hereafter $\mathrm{N}$ III/N II and NeIII/Ne II ratios, respectively). The extinction corrected map of the $\mathrm{N}$ III/N II ratio corresponding to the raster is shown in Fig. 3a. The circles in Fig. 3a indicate the location of the Quintuplet, the triangle indicates the location of the Pistol Nebula and the square indicates the position of the Arches cluster. The $\mathrm{N}$ III/N II ratio ranges from 0.33 to 2.52 . For the range of $n_{\mathrm{e}}$ derived from the $\mathrm{O}$ III $52 / 88$ ratio, the ratio of emissivities in the two nitrogen lines $\left(\epsilon_{57} / \epsilon_{122}\right)$ only changes from $\sim 6.3$ to $\sim 7.7$ (Rubin et al. 1994). Thus, in spite of the relatively low critical density of the N II line, the N III/N II ratio basically traces changes in the ionization fraction of $\mathrm{N}$ III relative to $\mathrm{N}$ II and not density variations. Although the $\mathrm{N}$ III/N II ratio shows a local maximum at the location of the Sickle, the absolute maximum is found towards the Pistol Nebula, close to the Quintuplet (although it is elongated towards the southwest). The N III/N II ratio shows a general trend to decrease as the distance to the Quintuplet cluster increases. This is illustrated in

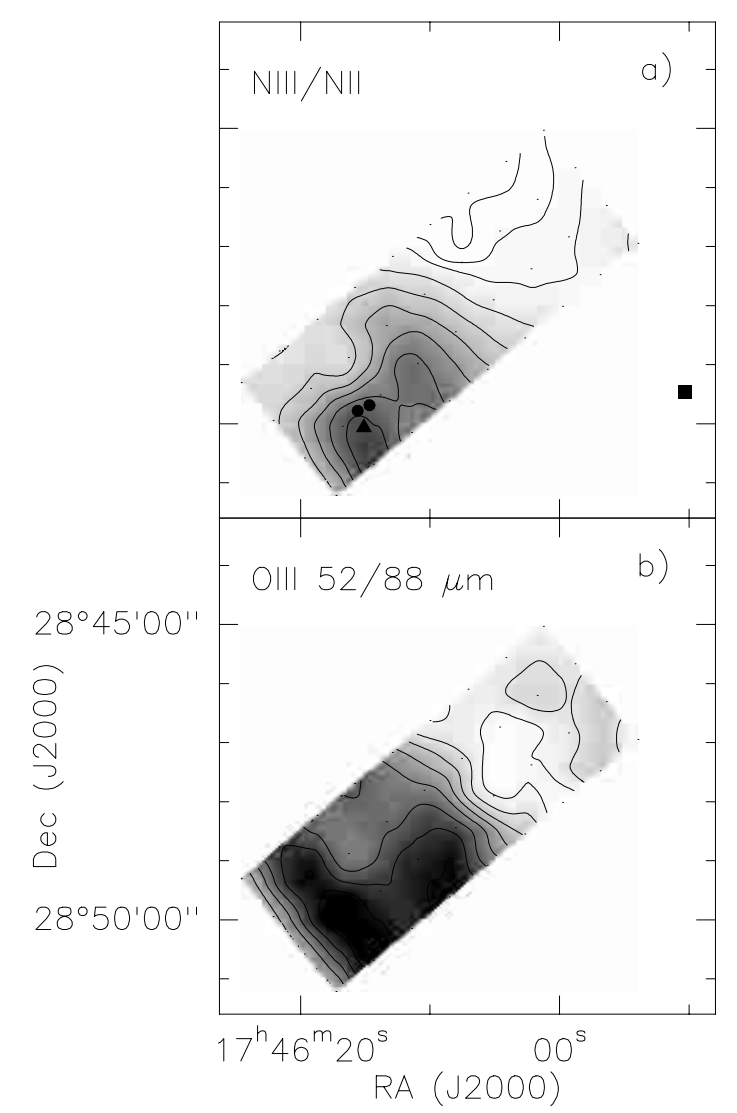

Fig. 3. a) N III $57 \mu \mathrm{m}$ to $\mathrm{N}$ II $122 \mu \mathrm{m}$ ratio map after correcting for $30 \mathrm{mag}$ of visual extinction (contour levels: 0.35, 0.45, and from 0.55 to 2.35 by 0.3 ). The circles indicate the position of the Quintuplet sources GCS 4 and GCS 3II and the triangle the location of the Pistol Nebula. The filled square indicates the position of the Arches cluster. b) O III 52 to $88 \mu \mathrm{m}$ line ratio corrected by $30 \mathrm{mag}$ of visual extinction. Contour levels: from 0.82 to 1.38 by steps of 0.08 .

Fig. 4, where we show all the N III/N II ratios as a function of their projected distance to the Quintuplet cluster. The N III/N II ratio shows a considerable dispersion which could be due to the difference between projected and actual distances and the possible minor contribution of other ionizing sources. It is remarkable that the degree of ionization toward sources like M1, M2, B4 and B5 located in the RAB, to the south and east of the Quintuplet, is similar to the ionization toward sources located in other directions but at similar distances from the cluster. This clearly points to the Quintuplet as the main ionization source of the RAB and not only of the Sickle and the Pistol Nebula.

The northwest part of the N III/N II map shows another gradient that does not point to the Quintuplet but towards other ionization source located in the direction of the Arches cluster. This cluster is believed to be the source of the ionization of the Arched Filaments (Colgan et al. 1996; Cotera et al. 2000a; Lang et al. 2001). In fact, the western-most part of the raster map covers the northern part of the E1 arched filament (for notation see for instance Lang et al. 2001). Figure 4 also shows this 
Table 4. Line ratios after correcting for $30 \mathrm{mag}$ of visual extinction. Numbers in parentheses are the rms errors of the last significant digit as derived from the errors in the Gaussian fits to the lines. To calculate line ratios we have taken into account the different beam sizes and considered extended emission.

\begin{tabular}{lllllll}
\hline & $\begin{array}{l}\text { S III } \\
\mathrm{R}(18 / 33)\end{array}$ & $\begin{array}{l}n_{\mathrm{e}}(\mathrm{S} \mathrm{III}) \\
\log \left(\mathrm{cm}^{-3}\right)\end{array}$ & Ne III/Ne II & $\begin{array}{l}\text { O III } \\
\mathrm{R}(52 / 88)\end{array}$ & $\begin{array}{l}n_{\mathrm{e}}(\mathrm{O} \text { III }) \\
\log \left(\mathrm{cm}^{-3}\right)\end{array}$ & N III/N II \\
\hline M1 & $0.66(2)$ & $<2$ & $0.148(7)$ & $0.88(8)$ & 1.96 & $0.9(2)$ \\
M2 & $0.697(13)$ & $<2$ & $0.094(3)$ & $1.04(5)$ & 2.17 & $0.68(6)$ \\
B1 & $0.82(2)$ & 2.48 & $0.134(6)$ & - & - & - \\
B2 & $0.59(2)$ & $<2$ & $0.109(5)$ & - & - & - \\
B3 & $0.55(2)$ & $<2$ & $0.047(7)$ & - & - & - \\
Q1 & $2.12(4)$ & 3.3 & $0.221(3)$ & - & - & - \\
Q2 & $3.36(7)$ & 3.78 & $0.19(2)$ & - & - & - \\
S1 & $0.86(3)$ & 2.70 & $0.133(4)$ & - & - & - \\
S2 & - & - & $0.119(2)$ & - & - & - \\
P & $1.71(2)$ & 3.18 & $0.296(4)$ & - & - & $0.68(7)$ \\
B4 & - & - & - & $0.96(8)$ & 2.07 & $0.45(7)$ \\
B5 & - & - & - & $0.79(10)$ & 1.84 & $0.31-2.52^{\mathrm{a}}$ \\
R & - & - & - & $0.78-1.43^{\mathrm{a}}$ & $1.81-2.56$ & 0 \\
\hline
\end{tabular}

${ }^{a}$ Maximum and minimum values. Typical errors lower than $10 \%$.

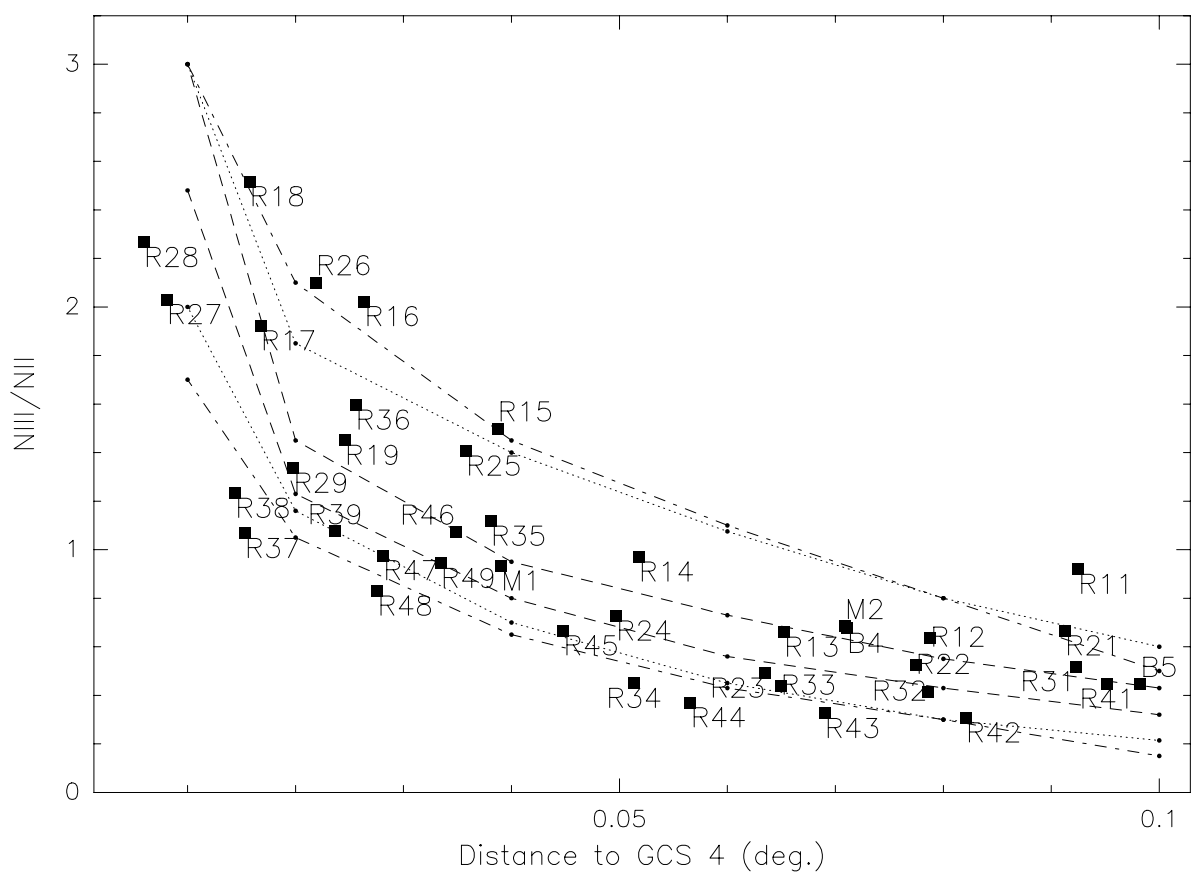

Fig. 4. N III $57 \mu \mathrm{m}$ to N II $122 \mu \mathrm{m}$ line intensity ratio as a function of the projected distance to the Quintuplet cluster source GCS 4 and comparison with models: Dot-dashed lines represent CLOUDY predictions for $Q(\mathrm{H})=10^{50.5} \mathrm{~s}^{-1}$ and Kurucz (1994) atmospheres with $35000 \mathrm{~K}$ (lower curve) and $36000 \mathrm{~K}$ (upper curve). Dashed lines are predictions for $Q(\mathrm{H})=10^{50.9} \mathrm{~s}^{-1}$ and CoStar atmospheres for $32600 \mathrm{~K}$ (lower curve) and $33300 \mathrm{~K}$ (upper curve). Dotted lines are the predictions for $Q(\mathrm{H})=10^{50.5} \mathrm{~s}^{-1}$ with CoStar atmospheres for $33300 \mathrm{~K}$ (lower curve) and $35500 \mathrm{~K}$ (upper curve).

effect. The N III/N II ratio measured for the raster points at distances larger than 0.05 show a systematic trend. For a given column $y$ of the raster, the $\mathrm{N}$ III/N II ratio decreases from the points in the first row of the raster (R1y) to the points in the fourth column (R4y). The effect is even more clear when one plots the N III/N II ratio derived for the first four columns of the raster as a function of their projected distance to the Arches cluster (see Fig. 5).
As for the NIII/NII ratio, the maximum of the Ne III/Ne II ratio is also found in the Quintuplet and Pistol Nebula sources. Figure 6 displays the NeIII/Ne II ratio as a function of projected distance to the Quintuplet. Although the number of available measurements is less than those of the nitrogen lines, the general behavior of the Ne III/Ne II ratio is similar to that of the N III/N II ratio, decreasing as distance to the Quintuplet increases. 


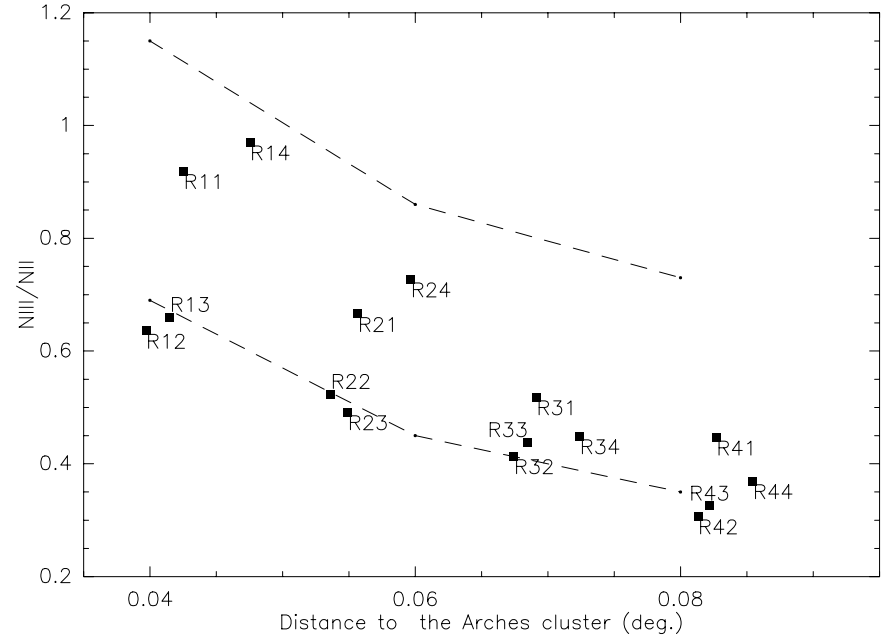

Fig. 5. N III/N II ratio derived from the first four columns of the raster map as a function of their projected distance to the Arches cluster and comparison with models predictions for using $Q(\mathrm{H})=10^{51.4} \mathrm{~s}^{-1}$ with CoStar atmospheres for $30400 \mathrm{~K}$ (lower curve) and $32600 \mathrm{~K}$ (upper curve).

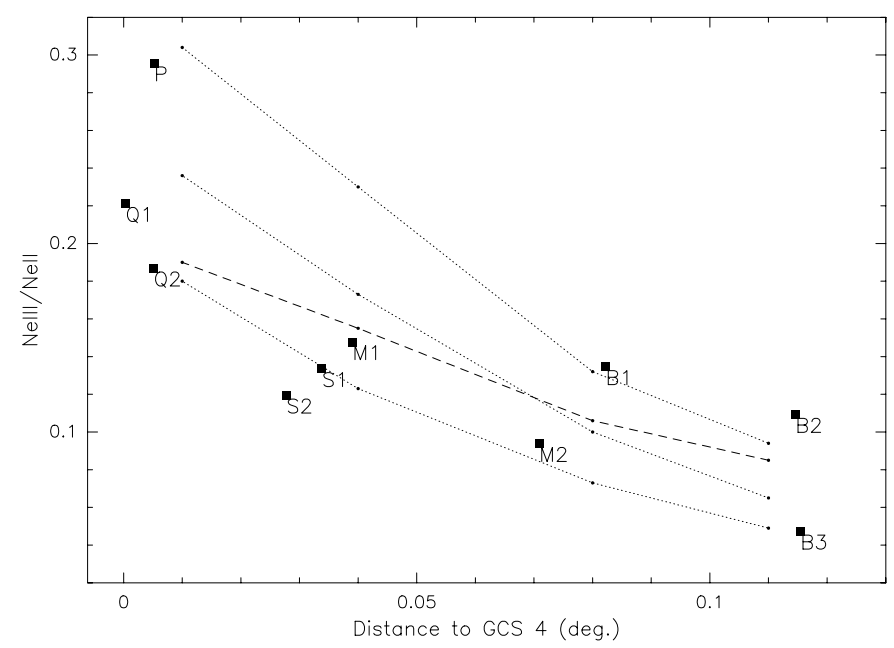

Fig. 6. NeIII/NeII ratio as a function of projected distance to the Quintuplet source GCS4. Dotted lines are model predictions for $Q(\mathrm{H})=10^{50.5} \mathrm{~s}^{-1}$ with CoStar atmospheres for 34500,35000 and $35500 \mathrm{~K}$ (from lower to upper curves). Dashed lines are the predictions for $Q(\mathrm{H})=10^{50.9} \mathrm{~s}^{-1}$ with CoStar atmospheres for $34500 \mathrm{~K}$.

However, the gradient in the NeIII/NeII ratio with distance is smaller than that exhibited by the $\mathrm{N}$ III/N II ratio $(\sim 3$ instead of $\sim 10)$. The critical densities of both the Ne III and the Ne II lines are $\gtrsim 2 \times 10^{5} \mathrm{~cm}^{-3}$. Thus, for the range of $n_{\mathrm{e}}$ derived for all the sources, the emissivities of both lines do not depend on $n_{\mathrm{e}}$, and like the N III/N II ratio, the NeIII/Ne II ratio traces the ionization structure.

\section{Origin of the ionization}

To study the large scale ionization structure we have compared the trend observed in the $\mathrm{N}$ III/N II and Ne III/Ne II ratios with the predictions of photoionization models considering the Quintuplet and the Arches clus- ters as the main ionizing sources. We have used CLOUDY version 90.04 (Ferland 1996) and the MICE interface developed by H. Spoon at the MPE to compute models for a grid of physical parameters. Namely, effective temperatures, $T_{\text {eff }}$, and Lyman continuum photons fluxes, $Q(\mathrm{H})$, of the ionizing sources. To define the shape of the incident continuum radiation we have used the models of stellar atmospheres developed by Kurucz (1994) and the Schaerer \& de Koter (1997) "CoStar" models, both for solar abundances. We have assumed nebular $\mathrm{N}$ and $\mathrm{Ne}$ abundances of $3 \times 10^{-4}$ and $2.8 \times 10^{-4}$, respectively, as derived by Rubin et al. (1995) for H II regions in the GCR. These abundances are a factor of $2-3$ higher than those measured for Galactic disk H II regions. We have checked with the Kurucz atmospheres that increasing the stellar metallicities in a factor of 3 changes the predicted lines ratios in less than $15 \%$. Therefore, enhanced metal abundances in the atmospheres does not affect significantly the results presented in the following sections. As a typical density of the ionized material we have used $10^{2.2} \mathrm{~cm}^{-3}$ as derived from the O III lines ratio.

\subsection{The Quintuplet cluster}

Since the Quintuplet cluster seems to dominate the ionization for most of the observed positions, we first considered this cluster as the ionizing source. We have used CLOUDY to estimate the size of the ionized region for the case in which the ionizing source is surrounded by material with an uniform $n_{\mathrm{e}}$ of $10^{2.2} \mathrm{~cm}^{-3}$. We assumed $Q(\mathrm{H})=10^{50.9} \mathrm{~s}^{-1}$ as derived by Figer et al. (1999) for the Quintuplet cluster and a $T_{\text {eff }}$ of $36300 \mathrm{~K}$ (B2 model of Schaerer \& de Koter). The predictions of CLOUDY for the size of the ionized region around the Quintuplet is of $\lesssim 6$ pc, much smaller than what is observed from the fine structure line ratios (which is $\sim 18 \mathrm{pc}$ assuming a distance to the Galactic center of $8.5 \mathrm{kpc}$ ). One can, however, explain the presence of Ne III and N III at such large distances from the Quintuplet with the $T_{\text {eff }}$ and $Q(\mathrm{H})$ derived for the Quintuplet if the material between the Quintuplet an the observed positions has a density of $\leq 10 \mathrm{~cm}^{-3}$. This is consistent with the ring, or almost empty bubble, morphology observed in the MSX images.

We have therefore used a simple model in which the ionized lines observed in our beam arise from clouds of density $10^{2.2} \mathrm{~cm}^{-3}$ located at a distance to the Quintuplet cluster equal to their projected distance on the sky (we assume a distance the GCR of $8.5 \mathrm{kpc}$ ). Under these conditions, one can characterize the effect of the radiation from a ionizing source on a cloud located at a distance $D$ using an ionization parameter $(U)$ defined as:

$U=\frac{Q(\mathrm{H})}{4 \pi D^{2} n_{\mathrm{e}} c}$

where $c$ is the velocity of light.

Following the models used by Simpson et al. (1997) for the Sickle and the Pistol Nebula, we have first computed the NIII/N II and NeIII/NeII ratios as a function 
of the distance of the clouds to the cluster (i.e. as a function of $U$ ) for $T_{\text {eff }}$ in the range of $34000-38000 \mathrm{~K}$ and $Q(\mathrm{H})$ in the range of $10^{49.5-50.5} \mathrm{~s}^{-1}$ with Kurucz (1994) atmospheres. We found that $Q(\mathrm{H})=10^{49.5} \mathrm{~s}^{-1}$ produces too small N III/N II ratios at large distances. On the opposite, $Q(\mathrm{H})=10^{50.5} \mathrm{~s}^{-1}$ can explain the derived ratios if $T_{\text {eff }}$ is in the range of $35000-36000 \mathrm{~K}$ (dot-dashed lines in Fig. 4). These values are in good agreement with those of Simpson et al. (1997) and indicate that our results extend to larger distances the results of Simpson et al. derived for the Sickle (see also Philipp et al. in prep). However, this model with the Kurucz atmospheres predicts Ne III/Ne II ratios which are a factor of $\sim 10$ smaller than the measured values. This is likely related to the well known Ne III/Ne II problem associated to the Kurucz atmospheres, which predict Ne III/Ne II ratios that are always much smaller than the measured ones. More recent model atmospheres give larger Ne III/Ne II ratios, much closer to the observed values (see e.g. Sellmaier et al. 1996).

We have tried to explain both the Ne III/Ne II and the $\mathrm{N}$ III/N II ratios consistently with similar parameters for the ionizing source using CLOUDY models with $Q(\mathrm{H})=$ $10^{50.9} \mathrm{~s}^{-1}$ using the stellar atmospheres of Schaerer \& de Koter. Figure 4 shows, as dashed lines, that the decrease of the $\mathrm{N}$ III/N II ratio with the distance is well explained with $T_{\text {eff }}=32600-33300 \mathrm{~K}$ (B3 and A2 CoStar models). The discrepancies between the observational results and the model are within a factor of $\sim 1.5$. This is quite reasonable in view of the simple model we use and the uncertainties introduced by its approximations. These are, mainly, the assumptions that projected distances are actual distances to the cluster and that there is not material in between the cluster and the observed sources (however, the model takes into account the attenuation of the radiation within the clouds). In addition, the model uses a single value of the $n_{\mathrm{e}}$ for all the positions. Figure 6 shows, also with dashed lines, that the predictions of this model for the Ne III/Ne II ratio are in better agreement with the ratios derived from the observations than those derived using the Kurucz atmospheres. However, the high $Q(\mathrm{H})$ value used to explain the $\mathrm{N}$ III/N II ratios predicts curves for the NeIII/NeII ratio as a function of distance that are rather flat. Moreover, the minimum $T_{\text {eff }}$ of $\sim 34500 \mathrm{~K}$ required in order to explain the Ne III/Ne II ratio is somewhat higher than the $T_{\text {eff }}(\sim 33000 \mathrm{~K})$ required to explain the $\mathrm{N}$ III/N II ratio.

For completeness, Figs. 4 and 6 also show, as dotted lines, the predictions for model calculations using CoStar atmospheres but with a slightly lower $Q(\mathrm{H})$ of $10^{50.5} \mathrm{~s}^{-1}$. Figure 4 shows that we can reproduce the observed behavior of the $\mathrm{NIII} / \mathrm{N}$ II ratio for $T_{\text {eff }}$ in the range of 33300 and $35500 \mathrm{~K}$. On the other hand, Fig. 6 shows that we also have a rather good agreement between the predictions and the measured NeIII/NeII ratio for $T_{\text {eff }} \sim 34500-35500 \mathrm{~K}$, which is similar to the $T_{\text {eff }}$ needed to explain the $\mathrm{N}$ III/N II ratio. In summary, the NIII/N II and NeIII/NeII ratios observed with ISO in the RAB region can be explained by assuming that the fine structure lines arise from clouds located at different distances from the Quintuplet cluster, which would be the main ionizing source. The required Lyman continuum photons flux of $10^{50.5-50.9} \mathrm{~s}^{-1}$ and effective temperatures of $\sim 35000 \mathrm{~K}$ are in good agreement with the estimations based on the stellar content of the cluster (see Figer et al. 1999).

\subsection{The Arches cluster}

As mentioned in Sect. 4.3 the observed gradient in the $\mathrm{N}$ III/N II ratio in the northwest region of the raster cannot be explained by the effect of the Quintuplet. In this section we will discuss the influence of the Arches cluster in the ionization of the Radio Arc material. Figure 5 shows the N III/N II ratio derived for the positions of the raster map which are closer to the Arches cluster as a function of their projected distance to this cluster. Figure 5 also displays, as long-dashed lines, the results of CLOUDY models computed with CoStar atmospheres, $Q(\mathrm{H})=10^{51.4} \mathrm{~s}^{-1}$ and $n_{\mathrm{e}}=10^{2.2} \mathrm{~cm}^{-3}$. The observed gradient in the $\mathrm{N}$ III/N II ratio is pretty well explained with a $T_{\text {eff }}$ in the range of $30400-32600 \mathrm{~K}$. These parameters are in agreement with those estimated for the Arches cluster (Morris et al. in prep; see also Lang et al. 2001). Therefore, we conclude that the observed N III/N II gradient to the southwest is likely produced by the Arches cluster and that the influence of the radiation from this cluster should be considered up to distances of $7-13 \mathrm{pc}$.

\subsection{The combined effect of both clusters}

We now consider the combined effects of the two clusters on the ionization of the whole region surrounding them. As discussed in Sect. 5.1, the N III/N II and Ne III/Ne II ratios are directly related to the ionization parameter $U$. Since the effective temperature of the radiation derived for both clusters are rather similar (see above), we have considered the most simple model to account for the combined effect of the two clusters.

As a first approximation one can estimate a "total ionization parameter" $\left(U_{\text {tot }}\right)$ as the sum of two different ionization parameters, one due to the Quintuplet $\left(U_{\mathrm{Q}}\right)$ and other due to the Arches cluster $\left(U_{\mathrm{A}}\right)$. Thus, for a cloud at distances $D_{\mathrm{Q}}$ and $D_{\mathrm{A}}$ from the Quintuplet and the Arches cluster, respectively, $U_{\text {tot }}$ will be given by:

$U_{\text {tot }}=U_{\mathrm{Q}}+U_{\mathrm{A}}=\frac{Q(\mathrm{H})_{\mathrm{Q}}}{4 \pi D_{\mathrm{Q}}^{2} n_{\mathrm{e}} c}+\frac{Q(\mathrm{H})_{\mathrm{A}}}{4 \pi D_{\mathrm{A}}^{2} n_{\mathrm{e}} c}$.

We have plotted in the upper panel of Fig. 7 some contours of equal $U_{\text {tot }}$ assuming a constant density of $n_{\mathrm{e}}=$ $10^{2.2} \mathrm{~cm}^{-3}$, a $Q(\mathrm{H})_{\mathrm{Q}}$ of $10^{50.9} \mathrm{~s}^{-1}$ and a $Q(\mathrm{H})_{\mathrm{A}}$ of $10^{51.4} \mathrm{~s}^{-1}$. The agreement of the iso- $U_{\text {tot }}$ curves with the contour map of the $\mathrm{N}$ III/N II ratio (shown with thick lines) is very good taking into account the simplicity of the model, which for instance, does not consider any shielding of the radiation. It reproduces the observed gradients 
towards the Arches and Quintuplet clusters. However, the model does not account for the local maxima found in the Sickle. The lower N III/N II ratios derived towards points located southward (in galactic latitude) of the Quintuplet which lie on the same iso- $U_{\text {tot }}$ curve than others located northward of the cluster should be due to the fact that $U_{\text {tot }}$ is more homogeneous in the LWS beam in the north of the cluster than in the south.

Furthermore, this simple model also reproduces the observed overall distribution of the warm dust. This is also illustrated in the upper panel of Fig. 7, where the iso- $U_{\text {tot }}$ contours are superposed on the $25 \mu \mathrm{m}$ band image taken by MSX. One can see that the morphology of the warm dust emission approximately follows the shape of the iso- $U_{\text {tot }}$ curves. The warm dust in the area of the Thermal Filaments is under the strong influence of the Arches cluster while the warm dust emission in the Sickle follows the shape of the iso- $U_{\text {tot }}$ curves that are dominated by the Quintuplet. The elliptical rather than circular distribution of the warm dust around the clusters, in particular around the Arches cluster, can be explained by the combined effect of both clusters. Even at large distances from the clusters ( $~ 215 \mathrm{pc}$ ) the distribution of the warm dust also seems to follow the shape of the iso- $U_{\text {tot }}$ curves, see for instance in Fig. 7 the $25 \mu \mathrm{m}$ dust emission in $(l, b) \sim(0.2,-0.15)$ or in $(l, b) \sim(0.05,-0.12)$. The agreement between the morphology of the warm dust emission with the iso- $U_{\text {tot }}$ curves strongly suggest a same origin for both the warm dust and the ionized gas (see next section).

\section{The Radio Arc bubble of warm dust}

The origin of the ring or bubble of warm dust it is not clear but the ISO data presented in this paper shows that the ionization of the gas is mainly produced by the Quintuplet instead of any source in the center of the ring. One unlikely possibility is that the hot dust observed in the RAB is not heated by the UV radiation from the clusters that explain the ionization structure (see Sect. 5.3). In this case, one would expect not to see a correlation between the warm dust and the ionization. We can check this possibility by comparing for instance the $\mathrm{O}$ III $88 \mu \mathrm{m}$ line intensity with the continuum emission from warm dust for different positions in the RAB. The intensity of this fine structure line for points "inside the ring" (M1, R19, R29; i.e. where the emission from warm dust is relatively weak, see Fig. 1) is lower than that for points like M2, B4 or B5 located on the edge of the ring. For instance, the average $\mathrm{O}$ III $88 \mu \mathrm{m}$ line fluxes for the points on the ring is $\sim 12 \times 10^{-18} \mathrm{~W} \mathrm{~cm}^{-2}$ while for those points inside the ring is $\sim 7 \times 10^{-18} \mathrm{~W} \mathrm{~cm}^{-2}$. From the IRAS HIRES 25 $\mu \mathrm{m}$ map (with a resolution of $66^{\prime \prime} \times 35^{\prime \prime}$ ) one obtains that the averaged intensity of the dust emission at this wavelength for the points on the edge of the ring, M2, B4 and $\mathrm{B} 5$, is $\sim 1500 \mathrm{M} \mathrm{Jy} \mathrm{sr}^{-1}$ and $\sim 750 \mathrm{M} \mathrm{Jy} \mathrm{sr}^{-1}$ for the points inside the ring (M1, R19, R29). Furthermore, towards the Sickle positions (R16-R46) the average O III $88 \mu \mathrm{m}$ line flux is $\sim 36 \times 10^{-18} \mathrm{~W} \mathrm{~cm}^{-2}$ while the $25 \mu \mathrm{m}$ intensity is $\sim 5000 \mathrm{M} \mathrm{Jy} \mathrm{sr}^{-1}$. Therefore, it seems that the ratio of the luminosity of the fine structure lines (ionized gas column density) to the warm dust emission is approximately constant for all positions in the RAB area. This constant line-to-continuum ratio shows that the warm dust should also be heated by the UV radiation from the cluster. The apparently lack of warm dust in the central part of the ring is just an effect of column density but not of UV radiation since the $\mathrm{N}$ III/N II ratio is higher for points like M1 inside the RAB, than for points at the edge of the RAB like $\mathrm{M} 2$.

The smaller column density of material towards the center of the ring is consistent with different geometries like a ring (or a cylinder with its axe close to the line of sight) and a shell or almost empty bubble. For a shell geometry with constant density and internal and external radius of 0.05 and 0.075 , respectively, the expected column density in the line of sight to the edge of the shell is a factor of $\sim 2$ larger than that in the line of sight towards the center of the shell. This is the typical ratio of the $\mathrm{O}$ III $88 \mu \mathrm{m}$ line or the $25 \mu \mathrm{m}$ dust emission between M2, B4, B5 at the edge of the shell and M1, R19, R29 at the center. Although this does not demonstrate that the observed structure has a shell morphology instead of a ring one, the data are consistent with a shell morphology (bubble) of dust heated, and gas ionized, by the UV radiation of the Quintuplet and the Arches cluster.

The geometrical center of the bubble is approximately located at $(l, b)=(0.16,-0$. 11$)$, over the NTFs. We have searched in the SIMBAD database for sources in the central area of the RAB without success. The closest sources to the geometrical center of the bubble are the near-IR sources 48 and 49 of Nagata et al. (1993) and our source M1 but no link between them and the RAB can be established. Somewhat further from the center there are other IR sources detected by Nagata et al. (1993) like Objects $43-46$ but they are late type M-stars candidates. However, close to this sources, there is also a luminous X-ray source: 1E 1742.9-2849 (represented as a filled circle in the lower panel of Fig. 7), with an X-ray luminosity of $\sim 8 \times 10^{34} \mathrm{erg} \mathrm{s}^{-1}$ (Predehl \& Trümper 1994, also recently detected by Chandra, Yusef-Zadeh priv. communication). It is $\sim 0.04(\sim 6 \mathrm{pc})$ far from the center of the shell and its position suggests that it could be responsible for the elongation of the maximum in the $\mathrm{N}$ III/N II ratio toward the southwest instead of being centered in the Quintuplet and the Pistol Nebula as expected from the models. There is another X-ray source inside the shell at a similar distance from its center: 1E 1743.1-2852 (triangle in the lower panel of Fig. 7).

It is remarkable that the diffuse hard X-rays $(2-10 \mathrm{keV})$ continuum emission observed by the ASCA satellite towards the GCR (Koyama et al. 1996) shows extended emission which covers an important fraction of the RAB. This would indicate that the RAB might be filled with hot gas that gives rise to the extended X-ray emission. One interesting prediction for the proposed scenario 


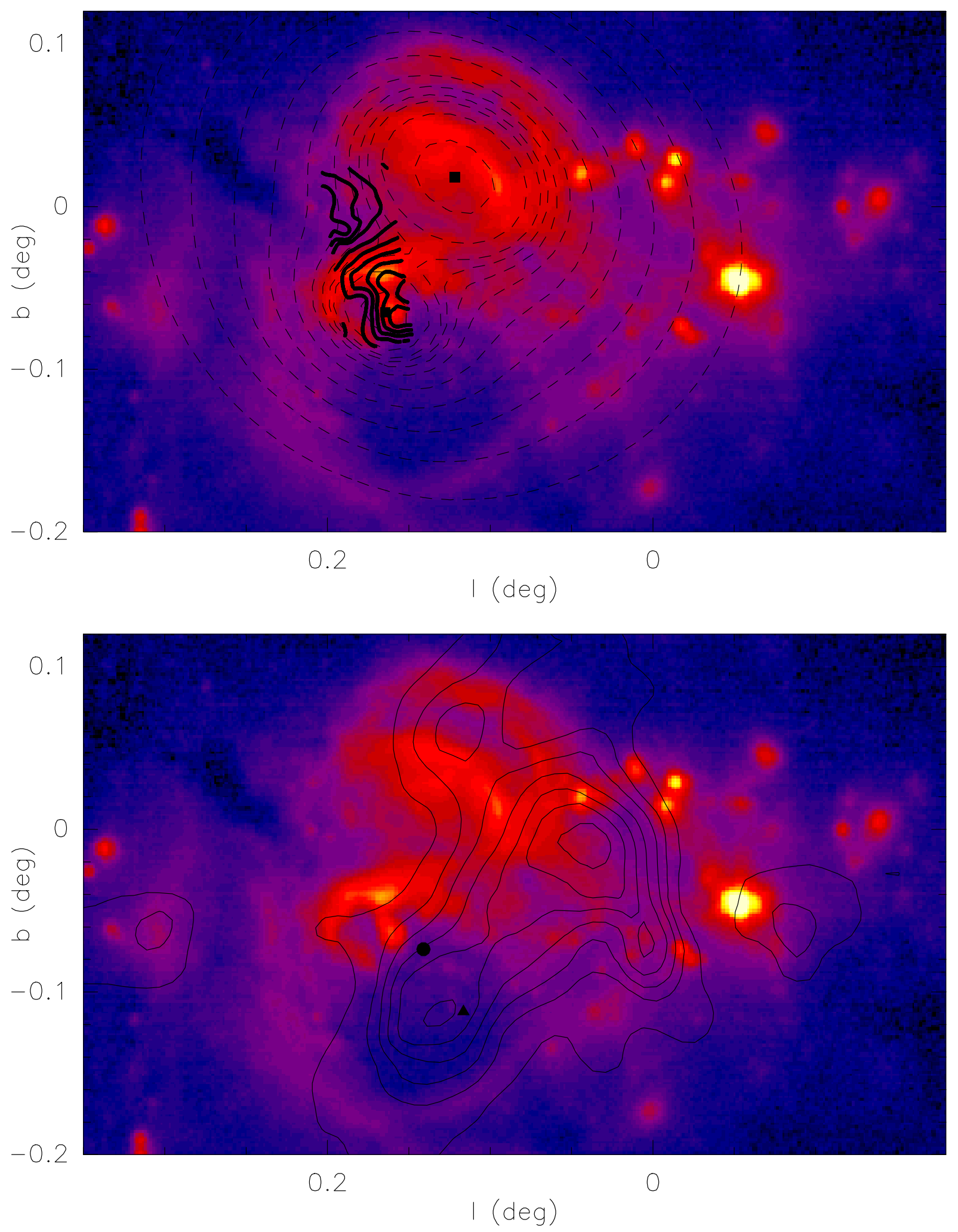

Fig. 7. Upper panel: contours of equal total ionization parameter (iso- $U_{\text {tot }}$ curves, see Sects. 5 and 6 ) overlaid on the $18-$ $25 \mu \mathrm{m}$ band image of MSX. The display is logarithmic from $10^{-6}$ to $2 \times 10^{-3} \mathrm{~W} \mathrm{~m}^{-2} \mathrm{sr}^{-1}$. The contour levels (log $\left.U_{\text {tot }}\right)$ are: $-0.5,-1,-1.15,-1.22,-1.3$, and from -1.4 to -2.15 by -0.15 . Thick contours are the N III/N II map as represented in Fig. 3 . Note that the model reproduces the observed gradients in the N III/N II ratio toward the Quintuplet and the Arches cluster (both represented as filled squares). In addition, the warm dust emission seems to follows the morphology of the iso- $U_{\text {tot }}$ curves. Lower panel: Fe $6.4 \mathrm{keV}$ line emission map of Koyama et al. (1996) overlaid in the 18-25 $\mu \mathrm{m}$ band MSX image. The contour levels of the $\mathrm{Fe} 6.4 \mathrm{keV}$ line map in units of $10^{-6}$ counts $/ \mathrm{s}^{-1} / 0.106 \mathrm{~min}^{2}$ are: 0.25 and from 0.4 to 1.1 by steps of 0.1 . Note that the Fe $6.4 \mathrm{keV}$ emission around $(l, b) \sim\left(0^{\circ} .13,-0^{\circ} .11\right)$ fills the bubble. The positions of $1 \mathrm{E} 1742.9-2849$ and $1 \mathrm{E} 1743.1-2852$ are shown by a solid circle and a triangle, respectively. 
of a bubble filled with hot gas is that the morphology of the $6.4 \mathrm{keV}$ line of neutral $\mathrm{Fe}$, which traces gas irradiated by hard X-rays (that is X-ray dominated regions - XDRs) should trace the walls of the bubble. The lower panel of Fig. 7 shows the Fe $6.4 \mathrm{keV}$ line emission map of Koyama et al. (1996) overlaid on the MSX image. The Fe $6.4 \mathrm{keV}$ line emission shows two maxima in the Sgr A neighborhood. One is located at $(l, b) \sim(0.04,-0.01)$, contouring the hard X-rays emission of the SgrA region while the other maximum is located at $(l, b) \sim(0.13,-0$. 11$)$ within the RAB. The Fe $6.4 \mathrm{keV}$ emission in the RAB peaks in between 1E1742.9-2849 and 1E 1743.1-2852, although closer to the last source. It clearly fills the bubble covering the region between the NTFs at $l \sim 0.18$ and the edge of the bubble at $l \sim 0$.08.

In summary, the RAB seems to be filled with hot gas emitting hard X-rays continuum which create a large XDR that is revealed by the $\mathrm{Fe} 6.4 \mathrm{keV}$ line emission. We speculate with the possibility that the X-ray sources might be related with the energetic events that created the bubbles of warm dust. Relative motions of the ISM and the $\mathrm{X}$-ray source would explain the off-center position of the $\mathrm{X}$-ray sources in the RAB. Considering the shape, the size of the bubble and the presence of the X-rays Fe lines, a supernova explosion is the most likely origin for the RAB.

\section{General implications}

As suggested by Martín-Pintado et al. 2000a, the picture arising from the comparison of the observed ionization and the model calculations is that the ISM in the Radio Arc region is highly inhomogeneous as indicated by the presence of bubbles filled with relatively low density ionized gas and hot gas emitting in X-rays. This structure allows the radiation from hot stars to reach large distances giving rise to extended low density ionized regions. The Quintuplet and the Arches cluster are the dominant sources of ionization in the Radio Arc region. Alternative ionization mechanisms (for instance magnetic effects) would play a minor role and should be restricted to smaller regions such as the Sickle.

This inhomogeneous structure seems to be a typical characteristic of the GCR ISM. In fact, this kind of structures has been invoked to explain the non-detection of hydrogen recombination lines and the detection of finestructure lines from ions like Ne II, S III or O III in a sample of clouds distributed all along the GCR (Martín-Pintado et al. 2000a; Rodríguez-Fernández et al. in prep.). On the other hand, inhomogeneities like shells and filaments are known to exist in other regions of the GCR like those revealed by the CO survey of Oka et al. (1998) or those found by Martín-Pintado et al. (1999) in the envelope of Sgr B2 which, like for the RAB, also seem to be correlated with the Fe $6.4 \mathrm{keV}$ line emission (see Martín-Pintado et al. 2000b).

\section{Conclusions}

We have presented ISO observations of selected fine structure lines in the Radio Arc in the Galactic center region. The analyzed lines are N III $57 \mu \mathrm{m}$, N II $122 \mu \mathrm{m}$, O III 52 and $88 \mu \mathrm{m}$, Ne III $15.6 \mu \mathrm{m}$, Ne II $12.8 \mu \mathrm{m}$ and S III 18.7 and $33.5 \mu \mathrm{m}$. The main results derived from these observations can be summarized as follows:

- The electronic densities derived from the O III 52 to $88 \mu \mathrm{m}$ ratio range between $10^{1.8}$ and $10^{2.6} \mathrm{~cm}^{-3}$. The electronic densities derived from the S III 18.7 to $33.5 \mu \mathrm{m}$ ratio in the smaller beam of the SWS are $\sim 10^{2} \mathrm{~cm}^{-3}$ for all the sources but those in the Quintuplet and Pistol Nebula which reach values of $\sim 10^{3.5} \mathrm{~cm}^{-3}$.

- After correcting for extinction, the N III/N II and the Ne III/Ne II ratios vary from position to position from 0.3 to 2.5 and from 0.05 to 0.30 , respectively. Both ratios exhibit a clear trend to decrease with increasing distance to the Quintuplet cluster. For some observed positions, the $\mathrm{N}$ III/N II ratio also shows a gradient that points towards the Arches cluster.

- Photoionization models confirm that the observed ratios and their trend with the distance to the clusters are consistent with the derived parameters for the clusters, that is $T_{\text {eff }}=32000-35000 \mathrm{~K}$, and a flux of Lyman continuum photons of $\sim 10^{51.4} \mathrm{~s}^{-1}$ and $\sim 10^{50.9} \mathrm{~s}^{-1}$ for the Arches and the Quintuplet cluster, respectively. Thus, the combined effects of both the Quintuplet and the Arches cluster completely dominate the ionization in the Radio Arc region. They create a large ionized region with a size of more than $30 \times 30 \mathrm{pc}^{2}$. Alternative ionization mechanisms (for instance magnetic effects or more hot stars) seems to play a minor role at large scales.

- A simple model of a total ionization parameter expected for the two clusters can explain not only the ionization structure but also the distribution of warm dust and, in particular, the elliptical rather than circular symmetry of some features like the Thermal Filaments. The warm dust seems to be correlated with the ionized gas and therefore also seems to be heated by the two clusters that ionize the gas.

- The effect of the radiation from the Quintuplet stars over large distances is at least in part due to the presence of a shell or an almost empty bubble (Radio Arc Bubble, RAB) of warm dust and ionized gas.

- The RAB is filled with hot gas emitting hard X-rays which creates a large X-ray dominated region (XDR) observed in the Fe $6.4 \mathrm{keV}$ line emission.

- The origin of the bubble it is not clear, but it could be related to a couple of $\mathrm{X}$-ray sources located $\sim 6 \mathrm{pc}$ off the shell center. Taking into account the size and shape of the bubble and the presence of the Fe X-rays lines, a supernova origin is certainly possible.

Acknowledgements. N. J. R.-F. acknowledges Consejería de Educación de la Comunidad de Madrid for a predoctoral 
fellowship. The authors acknowledge support by the Ministerio de Ciencia y Tecnología under grants 1FD97-1442 and PNE 014-2000-C. N. J. R.-F. thanks H. Spoon for his introduction to CLOUDY and MICE. MICE, SWS and the ISO Spectrometer Data Center at MPE are supported by DLR (DARA) under grants 50 QI 86108 and 50 QI 94023. The ISO Spectral Analysis Package (ISAP) is a joint development by the LWS and SWS Instrument Teams and Data Centers. Contributing institutes are CESR, IAS, IPAC, MPE, RAL and SRON. This research has made use of the SIMBAD database, operated at CDS, Strasbourg, France. This research made use of data products from the Midcourse Space Experiment. Processing of the data was funded by the Ballistic Missile Defense Organization with additional support from NASA Office of Space Science. The data were accessed by services provided by the NASA/IPAC Infrared Science Archive.

\section{References}

Catchpole, R. M., Whitelock, P. A., \& Glass, I. S. 1990, MNRAS, 247, 479

Chiar, J. E., Tielens, A. G. G. M., Whittet, D. C. B., et al. 2000, ApJ, 537, 749

Clegg, P. E., Ade, P. A. R., Armand, C., et al. 1996, A\&A, 315, L38

Colgan, S. W. J., Erickson, E. F., Simpson, J. P., Haas, M. R., \& Morris, M. 1996, ApJ, 470, 882

Cotera, A. S., Erickson, E. F., Colgan, S. W. J., et al. 1996, ApJ, 461, 750

Cotera, A., Colgan, S. W. G., \& Simpson, J. P. 2000, ISO beyond the peaks: The 2nd ISO workshop on analytical spectroscopy, ed. A. Salama, \& M. F. Kessler

Cotera, A. S., Simpson, J. P., Erickson, E. F., et al. 2000, ApJS, 129,123

de Graauw, T., Haser, L. N., Beintema, D. A., et al. 1996, A\&A, 315, L49

Draine, B. T. 1989, in Infrared Spectroscopy in Astronomy, ed. B. Kaldeich, ESA SP-290, 93

Egan, M. P., Shipman, R. F., Price, S. D., et al. 1998, ApJ, 494, L199

Ferland, G. J. 1996, Hazy, a Brief Introduction to Cloudy, University of Kentucky Department of Physics and Astronomy Internal Report

Figer, D. F., Najarro, F., Morris, M., et al. 1998, ApJ, 506, 384

Figer, D. F., McLean, I. S., \& Morris, M. 1999, ApJ, 514, 202

Glass, I. S., Moneti, A., \& Moorwood, A. F. M. 1990, MNRAS, $242,55 \mathrm{P}$

Güsten, R. 1989, IAU Symp. 136: The Center of the Galaxy, 136, 89

Kessler, M. F., Steinz, J. A., Anderegg, M. E., et al. 1996, A\&A, 315, L27

Koyama, K., Maeda, Y., Sonobe, T., et al. 1996, PASJ, 48, 249

Kurucz, R. 1994, Solar abundance model atmospheres for $0,1,2,4,8 \mathrm{~km} \mathrm{~s}^{-1}$. Kurucz CD-ROM No. 19. Cambridge, Mass.: Smithsonian Astrophysical Observatory, 1994, 19

Lang, C. C., Goss, W. M., \& Morris, M. 2001, AJ, 121, 2681

Leech, K., \& The SWS team 2001, SWS - The Short Wavelength Spectrometer, SAI/2000-008/Dc
Levine, D., Morris, M., \& Figer, D. 1999, in The Universe as Seen by ISO, ed. P. Cox, \& M. Kessler, ESA SP-427, 699

Lutz, D. 1999, in The Universe as Seen by ISO, ed. P. Cox, \& M. Kessler, ESA SP-427, 623

Martín-Pintado, J., Gaume, R. A., Rodríguez-Fernández, N., de Vicente, P., \& Wilson, T. L. 1999, ApJ, 519, 667

Martín-Pintado, J., Rodríguez-Fernández, N. J., de Vicente, P., et al. 2000a, ISO beyond the peaks: The 2nd ISO workshop on analytical spectroscopy, ed. A. Salama, M. F. Kessler, K. Leech, \& B. Schulz, ESA-SP 456, 111

Martín-Pintado, J., Vicente, P., Rodríguez-Fernández, N. J., Fuente, A., \& Planesas, P. 2000b, A\&A, 356, L5

Mezger, P. G., Duschl, W. J., \& Zylka, R. 1996, A\&AR, 7, 289

Moneti, A., Blommaert, J. A. D. L., Najarro, F., Figer, D., \& Stolovy, S. 1999, in The Universe as Seen by ISO, ed. P. Cox, \& M. Kessler, ESA SP-427, 723

Morris, M., \& Yusef-Zadeh, F. 1989, ApJ, 343, 703

Nagata, T., Woodward, C. E., Shure, M., Pipher, J. L., \& Okuda, H. 1990, ApJ, 351, 83

Nagata, T., Hyland, A. R., Straw, S. M., Sato, S., \& Kawara, K. 1993, ApJ, 406, 501

Nagata, T., Woodward, C. E., Shure, M., \& Kobayashi, N. 1995, AJ, 109, 1676

Odenwald, S. F., \& Fazio, G. G. 1984, ApJ, 283, 601

Oka, T., Hasegawa, T., Sato, F., Tsuboi, M., \& Miyazaki, A. 1998, ApJS, 118, 455

Okuda, H., Shibai, H., Nakagawa, T., et al. 1990, ApJ, 351, 89

Pauls, T., Downes, D., Mezger, P. G., \& Churchwell, E. 1976, A\&A, 46, 407

Pauls, T., \& Mezger, P. G. 1980, A\&A, 85, 26

Predehl, P., \& Trümper, J. 1994, A\&A, 290, L29

Rodríguez-Fernández, N. J., Martín-Pintado, J., Fuente, A., et al. 2001, A\&A, 365, 174

Rubin, R. H., Simpson, J. P., Lord, S. D., et al. 1994, ApJ, 420,772

Schaerer, D., \& de Koter, A. 1997, A\&A, 322, 598

Sellmaier, F. H., Yamamoto, T., Pauldrach, A. W. A., \& Rubin, R. H. 1996, A\&A, 305, L37

Serabyn, E., \& Güsten, R. 1991, A\&A, 242, 376

Serabyn, E., \& Morris, M. 1994, ApJ, 424, L91

Serabyn, E., Shupe, D., \& Figer, D. F. 1998, Nature, 394, 448

Schultheis, M., Ganesh, S., Simon, G., et al. 1999, A\&A, 349, L69

Simpson, J. P., Colgan, S. W. J., Cotera, A. S., et al. 1997, ApJ, 487, 689

Swinyard, B. M., Clegg, P. E., Ade, P. A. R., et al. 1996, A\&A, 315, L43

Timmermann, R., Genzel, R., Poglitsch, A., et al. 1996, ApJ, 466,242

Yusef-Zadeh, F., \& Morris, M. 1987a, ApJ, 320, 545

Yusef-Zadeh, F., \& Morris, M. 1987b, ApJ, 322, 721

Yusef-Zadeh, F., \& Morris, M. 1987c, AJ, 94, 1178

Yusef-Zadeh, F., Morris, M., \& Chance, D. 1984, Nature, 310, 557

Yusef-Zadeh, F., Morris, M., \& van Gorkom, J. H. 1987, in The Galactic Center, ed. B. C. Backer, AIP Conf. Proc., 155, 190 